\title{
Electrophysiological Analysis Shows Dizziness as the First Symptom in Human T Cell Lymphotropic Virus Type-Associated Myelopathy/Tropical Spastic Paraparesis
}

\author{
Ludimila Labanca,,2 Ana Lúcia Borges Starling, ${ }^{1-3}$ Silvio Roberto de Sousa-Pereira, \\ Luiz Cláudio Ferreira Romanelli, ${ }^{2,3}$ Anna Bárbara de Freitas Carneiro-Proietti, 2,3 \\ Lucas Novaes Carvalho, Daniele Rosa Fernandes, and Denise Utsch Gonçalves ${ }^{1,2}$
}

\begin{abstract}
Dizziness is a symptom in human $\mathrm{T}$ cell lymphotropic virus type-associated myelopathy/tropical spastic paraparesis (HAM/TSP) and may occur due to vestibulospinal tract dysfunction. This tract can be assessed by an electrophysiological test called vestibular-evoked myogenic potential (VEMP). The aim was to correlate the result of VEMP generated by acoustic stimuli and dizziness in individuals with human $\mathrm{T}$ cell lymphotropic virus type 1 (HTLV-1)-asymptomatic infection and HAM/TSP. VEMP was recorded from the sternocleidomastoid muscle of 60 HTLV-1-negative adults (60 8 years) and 60 individuals infected with HTLV-1, 30 being asymptomatic ( $59 \pm 8$ years) and 30 with HAM/TSP (59 \pm 8 years). In all groups, $90 \%$ of the participants were women. VEMP was generated by acoustic stimuli (short tone bursts), with an intensity of $118 \mathrm{dBHL}$ and band-pass filter from $10 \mathrm{~Hz}$ to $1,500 \mathrm{~Hz}$, and presented 200 stimuli at a frequency of $1,000 \mathrm{~Hz}$ with a record time of $60 \mathrm{~ms}$. Of $60 \mathrm{HTLV}-1$-negative individuals, 14 (23\%) reported dizziness; VEMP was normal in all. In the HTLV-1-asymptomatic group, 11(37\%) complained of dizziness ( $p=0.31)$; VEMP was altered in four $(40 \%)$ subjects with dizziness and in one $(5 \%)$ without dizziness $(p=0.00)$. In the group with HAM/TSP, dizziness was reported by $17(57 \%)$ subjects $(p=0.002)$; VEMP was altered in $11(64 \%)$ with dizziness and in $5(38 \%)$ without dizziness $(p=0.15)$. Dizziness without an apparent etiology in HTLV-1-asymptomatic carriers deserves attention in terms of a possible subclinical spinal cord involvement that can be clarified through spinal electrophysiological tests. Damage of the vestibulospinal tract seems to occur in the early stages of HAM/TSP.
\end{abstract}

\section{Introduction}

$\mathbf{H}$ UMAN T CELL LYMPHOTROPIC VIRUS type 1 (HTLV-1) causes inflammatory changes in the spine. ${ }^{1-3}$ HTLV-1associated myelopathy/tropical spastic paraparesis (HAM/ TSP) is a disease of slow and progressive evolution that predominantly affects the anterior thoracic spinal cord, although the entire neuraxis can be damaged, including fibers that influence the control of muscle tone, such as the cerebellum, brainstem, and vestibulospinal tracts. ${ }^{4,5}$ Thus, it is expected that the reflexes related to body balance may be altered in HAM/TSP. ${ }^{4}$ Actually, frequent complaints of dizziness among individuals with HAM/TSP has been re- ported $^{6,7}$ and damage of the vestibulospinal pathway was identified through vestibular-evoked myogenic potential (VEMP) as a precocious electrophysiological alteration of individuals who afterward developed HAM/TSP. ${ }^{6,8,9}$ However, dizziness as a clinical manifestation has not yet been correlated with the electrophysiological alterations of the vestibulospinal pathway and had not yet been analyzed as a prognostic marker of HAM/TSP.

The aim of the present study was to compare the complaints of dizziness with the results of VEMP generated by acoustic stimuli in individuals with HTLV-1-asymptomatic infection and individuals with HAM/TSP, using a matched group by age and gender of healthy and HTLV-1-seronegative individuals.

\footnotetext{
${ }^{1}$ Tropical Medicine Post Graduate Program, Faculty of Medicine, Federal University of Minas Gerais, Belo Horizonte, Minas Gerais, Brazil.

${ }^{2}$ Interdisciplinary HTLV Research Group (GIPH), Belo Horizonte, Minas Gerais, Brazil.

${ }^{3}$ Minas Gerais State Center of Hematology and Blood Transfusion (Fundação Hemominas), Belo Horizonte, Minas Gerais, Brazil.

(c) Ludimilia Labanca et al. 2015; Published by Mary Ann Liebert, Inc. This Open Access article is distributed under the terms of the Creative Commons Attribution Noncommercial License ( $<$ http://creativecommons.org/licenses/by-nc/4.0/ $>$ ) which permits any noncommercial use, distribution, and reproduction in any medium, provided the original author(s) and the source are credited.
} 


\section{Materials and Methods}

\section{Ethical aspects}

Approval was obtained by the Ethics Committee of the Federal University of Minas Gerais, number 0437.0.203.00010, and the Ethics Committee of the Hemominas Foundation, number 290. All participants gave voluntary written consent and declared they were aware of the procedures and their freedom to participate.

\section{Subjects}

The Interdisciplinary HTLV Research Group (GIPH) studies the natural history, the clinical manifestations, and the epidemiological aspects of HTLV infection. ${ }^{10,11}$ This group has been following individuals infected with HTLV since 1997 in the state of Minas Gerais, Brazil, in an open cohort. As part of the cohort, over 637 HTLV-infected and 232 noninfected individuals were enrolled. ${ }^{11}$

The present study is a transversal evaluation of 120 participants in the GIPH cohort. ${ }^{2,11}$ The participants were randomly selected from the list of individuals enrolled in the cohort. The HTLV-1-seropositive participants fulfilled the criteria of either being asymptomatic carriers or having HAM/TSP. ${ }^{12}$ The individuals were invited to participate by telephone for the HTLV-1-seropositive group and by personal contact before blood donation for the HTLV-1seronegative group.

The participants were 60 healthy blood donors and 60 infected with HTLV-1. The latter group consisted of two subgroups, according to the Expanded Disability Status Scale $(\text { EDSS })^{13}$ and OSAME scale ${ }^{14}: 30$ HTLV-1-asymptomatic carriers, from a neurological point of view (EDSS and OSAME - 0 in both scales) and 30 with a definite diagnosis of HAM/TSP (EDSS and OSAME greater than 2 on both scales). The 60 individuals noninfected with HTLV-1 were matched by gender and age with the individuals of the groups infected with HTLV-1.

\section{HTLV-1 diagnosis}

All the participants were submitted to serological screening for blood-borne pathogens, regularly applied in blood centers. The HTLV-1 infection in blood was based on two positive examinations using different samples. The individuals with a reactive HTLV screening test (enzyme-linked immunosorbent assay, ELISA, Bio-Rad, USA) were tested by Western blot (WB-MP Diagnostics, Singapore); those with any other positive results in the screening tests were excluded. ${ }^{10}$

\section{Study design}

The study was a sectional analysis of the GIPH cohort. The risk factor was HTLV-1 infection and the dependent variables were manifestations of dizziness and VEMP analysis. All participants underwent a medical interview concerning their general health status and otoneurological manifestations and were evaluated by the otolaryngologist, neurologist, and audiologist and submitted to specific tests to exclude any clinical cause of dizziness. ${ }^{15-17}$

All the participants underwent VEMP and all the individuals with dizziness were submitted to a clinical and otoneurological examination along with specific examinations in order to clarify the causes related to peripheral labyrinth disease and related to neurological, psychiatric, cardiovascular, endocrinological, and orthopedic diseases that may present dizziness. ${ }^{16}$

\section{Vestibular-evoked myogenic potential}

VEMP testing has been used to evaluate diseases related to the peripheral vestibular system, mainly the saccule and its afferents. ${ }^{18}$ This evoked potential is activated by either sound or galvanic stimulation and both stimuli trigger the vestibulospinal myogenic reflex. ${ }^{9,18}$ The pathways of VEMP activated by sound are the saccular macula in the internal ear, the inferior vestibular nerve, the medial vestibular nucleus in the brainstem, the vestibular spinal descending pathways, and finally the motor neurons of the ipsilateral sternocleidomastoid muscle. ${ }^{19}$ As the neural pathway includes the vestibulospinal tract, this testing can also be used to evaluate the integrity of the spinal cord. ${ }^{20}$

The VEMP response consists of two waves of reversal polarity at $13 \mathrm{~ms}$ and $23 \mathrm{~ms}^{21}$ The first wave is positive (P13) followed by a second negative wave (N23). ${ }^{21}$ The changes of the amplitude are the most reliable measure to evaluate diseases of the internal ear ${ }^{22}$ and the prolonged latency, mainly of P13, can be found in central disturbances. ${ }^{23}$

The use of VEMP in the evaluation of patients with HAM/ TSP has previously been described. ${ }^{6,8,9}$ In the present study, high-intensity sound was used to activate the reflex, and the response was recorded by surface electromyography electrodes on the sternocleidomastoid muscle. ${ }^{18,19,21}$ The eletromyographic (EMG) activity was recorded from the upper half of the sternocleidomastoid (SCM) muscle using surface electrodes, with a reference on the upper sternum and a ground electrode on the forehead. ${ }^{9,20}$ During each recording session, in seated position, the subject was instructed to rotate the head toward the contralateral side of the tested ear to keep the SCM muscle under tension (Fig. 1). VEMP was generated by acoustic stimuli (short tone bursts), with an intensity of $118 \mathrm{dBHL}$ and band-pass filter from $10 \mathrm{~Hz}$ to $1,500 \mathrm{~Hz}$, was presented 200 stimuli at a frequency of $1,000 \mathrm{~Hz}$ with a record time of $60 \mathrm{~ms}$, and the electromyographic signals were amplified (model MASBE/ActPlus, Contronic, BR). The response was recorded from the SCM muscle on each side. The examination was replicated to confirm the eletrophysiolological origin.

\section{Data analysis}

The analysis of VEMP results was masked and was done by two examiners in a blind way. The measured parameters were the peak latency of the two waves P13-N23. ${ }^{19,21}$ The accepted variation of normality was two standard deviations of P13-N23 values of the uninfected group, which was latency $\leq 15 \mathrm{~ms}$ for P13 and $\leq 26 \mathrm{~ms}$ for N23.

The results of VEMP were classified as normal (Fig. 2A) or abnormal (Fig. 2B and C). The abnormal VEMP showed either prolonged latency (Fig. 2B) or undetectable response (Fig. 2C). The results of VEMP were compared with the complaint of dizziness for each group (HTLV-1 asymptomatic, HAM/TSP, and healthy individuals).

The statistical analysis was performed using the Statistical Package for Social Sciences, version 18.0 for Windows, SPSS 
FIG. 1. Technical execution of vestibular-evoked myogenic potential (VEMP): ear phones and electrode position. GE, ground electrode; AE, activity electrode on sternocleidomastoid muscle; R, reference electrode on sternum.

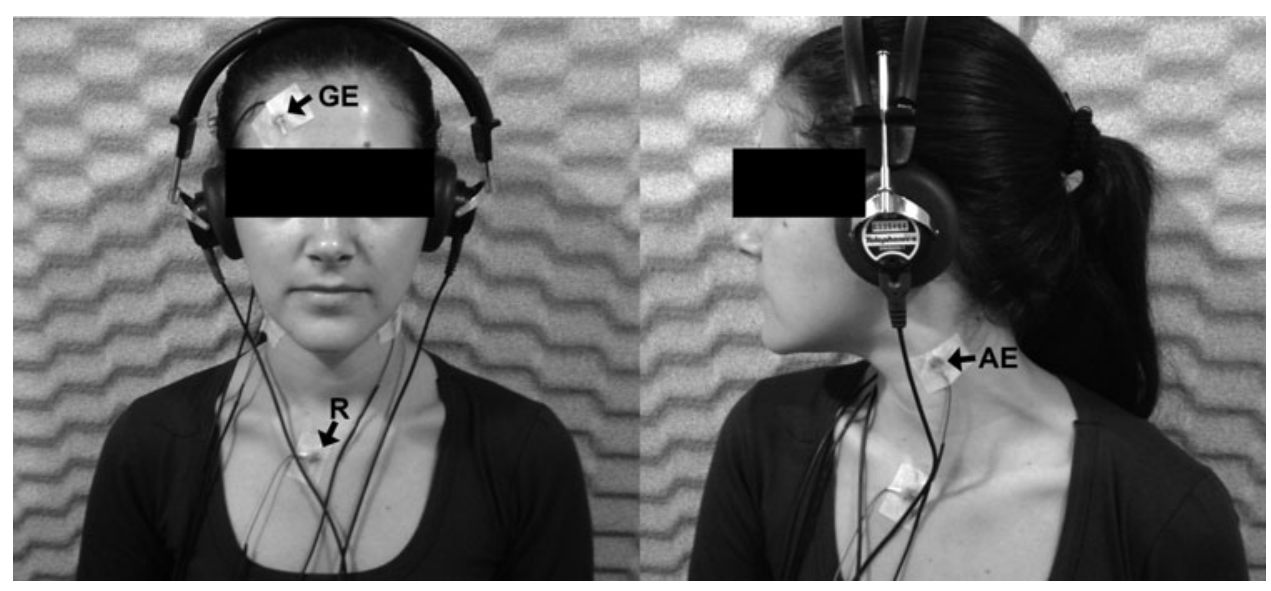

(Chicago, IL). The between-groups comparisons for continuous variables were made through the Mann-Whitney test or $t$-test. The between-groups comparisons for categorical variables were made through the Qui-square Pearson test or Fisher test when samples with small frequencies were used. In the cross-analysis of correlations, a significance level of $5 \%$ (error type I) was considered and a power of $80 \%$ (type II error). Aiming at the magnitude of the association between exposure to the risk factor and the dependent variables, the prevalence ratio (PR) was calculated. The confidence interval (95\%) was calculated in order to evaluate the sample variability.

\section{Results}

Among the 60 individuals uninfected with HTLV-1 (controls), the mean age was $60 \pm 8$ (SD) years. Among the HTLV-1-infected individuals, the mean age was $59 \pm 8$ years for the 30 asymptomatic carriers and $59 \pm 8$ years for the 30 with HAM/TSP $(p=0.733)$. In all groups, $90 \%$ of the participants were women.

In the uninfected group, VEMP was found to have the same pattern of wave for all individuals (Fig. 2A). In the
HTLV-1-infected group, the most common pattern was prolonged latency for the asymptomatic group and undetectable response and prolonged latency for the HAM/TSP group.

In the HTLV-1 asymptomatic group, VEMP was abnormal in $5 / 30(17 \%)$ participants. The characteristic of abnormal response was prolonged latency. In the HAM/TSP group, VEMP was abnormal in 16/30 (53\%) participants. A prolonged response was observed in 12/16 (75\%) participants and an undetectable response in 4/16 (25\%) participants.

Figure 3 shows the frequency of dizziness in each group. Of 60 HTLV-1-uninfected individuals, 14 (23\%) reported dizziness; VEMP was normal in all. In the HTLV-1-asymptomatic group, $11 / 30(37 \%)$ complained of dizziness $(p=0.31)$; VEMP was altered in 4/11 (40\%) subjects with dizziness and in $1 / 19(5 \%)$ subjects without dizziness $(p=0.00)$. In the group with HAM/TSP, dizziness was reported by $17 / 30$ (57\%) subjects $(p=0.002)$; VEMP was altered in $11 / 17(65 \%)$ subjects with dizziness and in $5 / 13(38 \%)$ subjects without dizziness $(p=0.15)$.

Figure 4 shows the comparison of VEMP latency among the groups with and without dizziness. The participants with
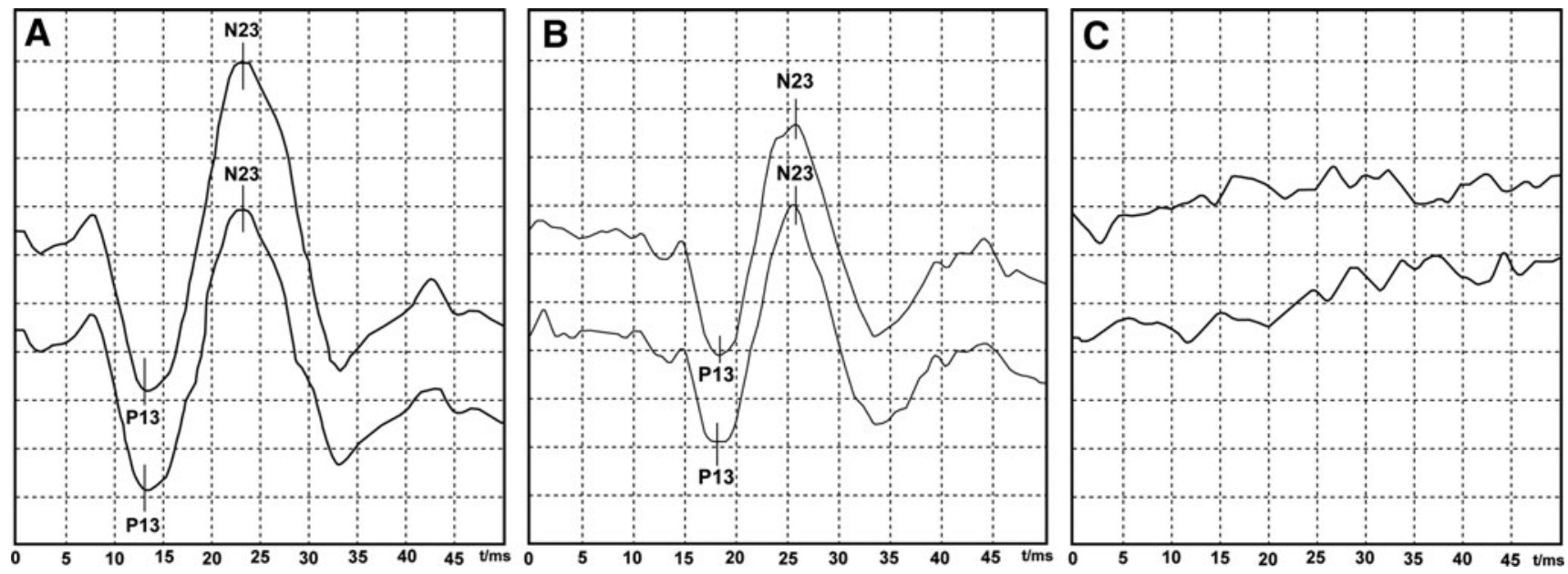

FIG. 2. Examples of tracings obtained by vestibular-evoked myogenic potential (VEMP) recorded from the sternocleidomastoid muscle. (A) VEMP with normal latency of an uninfected individual. (B) VEMP with prolonged latency of an individual with an apparent asymptomatic human T cell lymphotropic virus type 1 (HTLV-1) infection. (C) VEMP with undetectable response of an individual with HAM/TSP. t, time; ms, milliseconds 


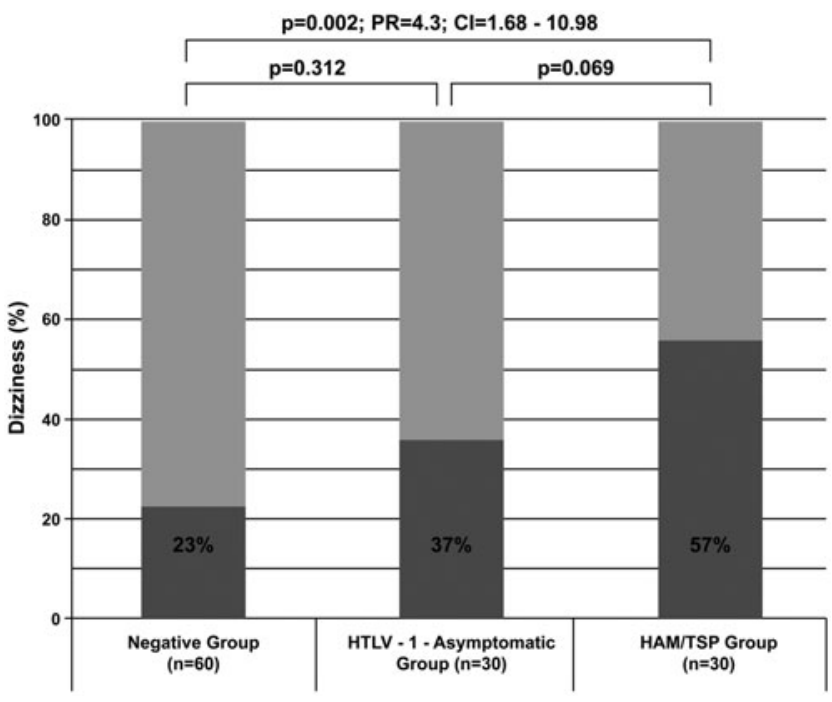

FIG. 3. Dizziness among the groups: HTLV-1-asymptomatic carrier, HAM/TSP, and uninfected individuals. $N=120$. Light gray box, absence of dizziness; dark gray box, presence of dizziness; $n$, number of participants; PR, prevalence ratio; $\mathrm{CI}$, confidence interval; $p, p$-value (ChiSquare test or Fisher's Exact test).

undetectable response were not plot in the figure and the statistical significance was reached considering the results in which latency was record. The results show that the latency of VEMP was higher in HTLV-1-asymptomatic $(p=0.02)$ and HAM/TSP groups with dizziness $(p=0.03)$.

\section{Discussion}

In HTLV-1-endemic areas, HTLV-1 seroprevalence increases with age and is higher in women than in men. ${ }^{1,24}$ The increase of prevalence with age may be explained by the accumulation of seroconversions over the lifetime; the higher prevalence in females may be due to a more efficient male-tofemale transmission during sexual intercourse. Hormonal effects may also play a role in female susceptibility. ${ }^{24}$

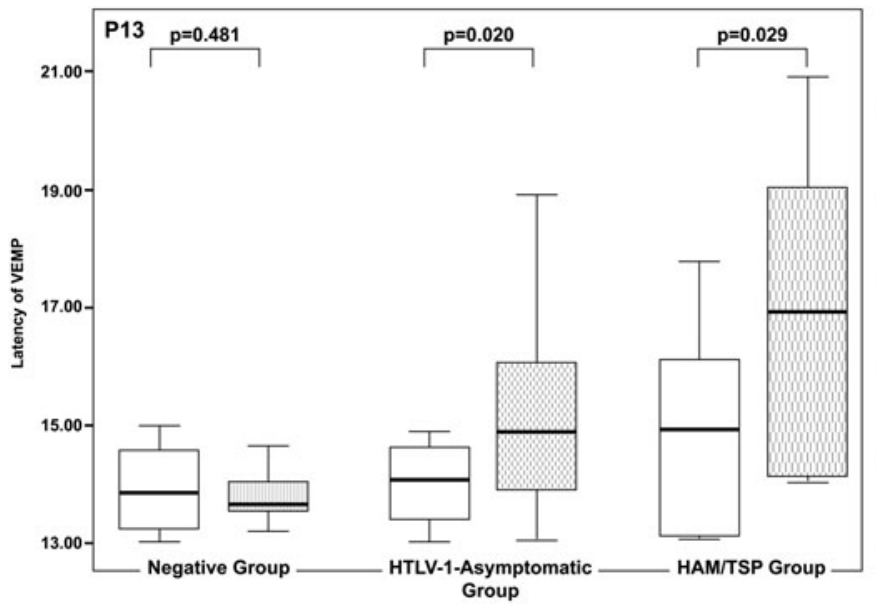

In the present study, the frequency of dizziness was approximately four times higher in those with HAM/TSP compared to the HTLV-1-seronegative individual. Interestingly, for the HTLV-1-asymptomatic carriers, the complaint of dizziness was similar in frequency when compared to the general population in which this symptom is mentioned by $20 \%$ to $30 \%$ of individuals aged 60 years. $^{25}$

The normal balance takes for granted the correct interaction of the vestibular mechanisms with the visual perception and with the proprioception. ${ }^{20}$ The lesion or dysfunction of any of these mechanisms can trigger disturbances in balance control. ${ }^{16,17}$ Dizziness presented as vertigo is usually related to the impairment of unilateral peripheral vestibular pathways while instability is more commonly related to direct or indirect damage of CNS vestibular projections, including the vestibulospinal tracts. ${ }^{17}$ Characteristically, HAM/TSP affects the motor tracts and tracts nearby, including the vestibulospinal and reticulospinal tracts. ${ }^{26}$

The complaint of dizziness was shown to be mentioned early in the spectrum of the neurological symptoms related to HAM/TSP. ${ }^{7}$ In the present study, the electrophysiological examination of the vestibulospinal tract through VEMP showed altered responses in the HTLV-1-infected individuals with dizziness either with HAM/TSP or asymptomatic carriers. The stronger statistical association between the complaint of dizziness and the altered VEMP in the group of HTLV-1-asymptomatic carriers emphasizes the value of this symptom as a possible neurological manifestation related to incipient damage of the vestibulospinal tract.

Individuals with HAM/TSP complain more frequently of dizziness than individuals with other nontraumatic myelopathies, as, for example, in schistosomiasis myeloradiculopathy. ${ }^{27}$ The difference in the pathophysiology explains the higher occurrence of dizziness in HAM/TSP, while the damage of the motor tract in the schistosomiasis myeloradiculopathy is due to the parasite's eggs obstruction of the inferior spinal vascular system, which causes a well-delimitated inflammation. ${ }^{28}$ In HAM/TSP, the damage of the spinal cord is due to the predominance of inflammatory cytokines and chemokines in the spinal cord causing damage to the motor tract and nearby areas. ${ }^{24}$

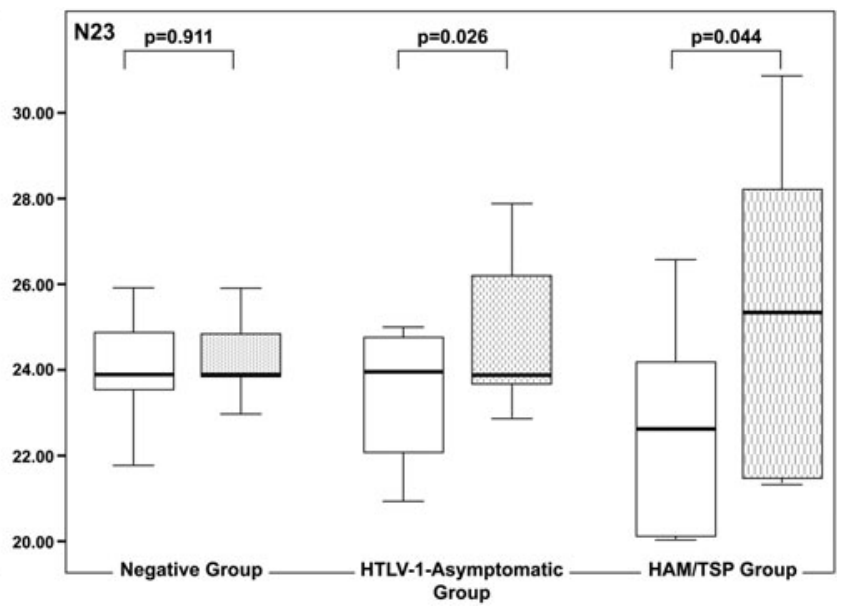

FIG. 4. Comparison of the vestibular-evoked myogenic potential (VEMP) latency among the groups: HTLV-1 uninfected, HTLV-1-asymptomatic carrier, and with HAM/TSP. $N=120$. Blank box, individuals without dizziness; dashed box, individuals with dizziness; $p, p$-value (Mann-Whitney test). 
Although the focus of the damage is the inferior spinal cord, all the neuroaxis is affected. ${ }^{23}$

The morphological alterations related to HAM/TSP allow speculative explanations about the cause of the dizziness. ${ }^{5,24}$ $\mathrm{HAM} / \mathrm{TSP}$ is an inflammatory myelopathy that predominates at the descending spinal pathways. The damage of the reticulospinal and the vestibulospinal tracts, both related to balance control, may explain the high frequency of dizziness in individuals with HAM/TSP. ${ }^{6}$ Subclinical alterations can occur in apparently asymptomatic HTLV-1-infected populations. ${ }^{9}$ This finding was confirmed by the present study.

VEMP has previously been shown to be more altered in patients with HAM/TSP when compared to the HTLV-1asymptomatic carriers, although the correlation with dizziness had not been set. ${ }^{8,9}$ The delay in the vestibulospinal reflex may be the explanation for this finding. The integrity of corticospinal tract in individuals with HAM/TSP was scrutinized using the motor-evoked potentials elicited by transcranial magnetic stimulation and the results were consistent with a delay in the motor responses. ${ }^{29}$ VEMP is an objective test and the association of altered results with the complaint of dizziness may be an indication of early neurological damage caused by HTLV-1. ${ }^{6,8,9}$ The next question to be answered concerns the value of an altered VEMP in an HTLV-1-asymptomatic carrier as a prognostic sign of HAM/ TSP development.

About 5\% of the individuals infected with HTLV-1 develop HAM/TSP, usually after the fourth decade. Numerous studies have been looking for a immunological or clinical prognosis biomarker. ${ }^{2,30}$ The HAM/TSP diagnosis has usually been late and has been made when the person gradually evolves to symmetrical paraparesis of the lower limbs with signs of pyramidal tract involvement. ${ }^{3}$ The first symptoms have been related to urinary and sexual problems. ${ }^{12}$ Based on the present study, dizziness may precede the described initial symptoms.

\section{Conclusions}

Dizziness without an apparent etiology in HTLV-1asymptomatic carriers deserves attention in terms of a possible subclinical spinal cord involvement and should be clarified through spinal electrophysiological tests. Damage to the vestibulospinal tract seems to occur in the early stages of HAM/TSP.

\section{Acknowledgments}

This work was supported by the CNPQ, FAPEMIG, Fundação Hemominas, Faculdade de Medicina-Programa de Pós Graduação em Infectologia e Medicina Tropical da FM/ UFMG, CAPES. The authors would also like to thank the remaining participants of the GIPH cohort study for their support.

\section{Author Disclosure Statement}

No competing financial interests exist.

\section{References}

1. Cervilla JO, Cartier LR, and Garcia LF: Brain and spinal cord magnetic resonance imaging in spastic paraparesis associated to human T-lymphotropic virus. Rev Méd Chile 2006;134:1010-1018.

2. Romanelli LCF, Caramelli P, Martins ML, et al.: Incidence of HTLV-1 associated myelopathy (HAM/TSP) in a long-term prospective cohort study of initially asymptomatic individuals in Brazil. AIDS Res Hum Retroviruses 2013;29(9):1199_ 1202.

3. Iwasaki Y: Human $\mathrm{T}$ cell leukemia virus type 1 infection and chronic myelopathy. Brain Pathol 2011;3:1-10.

4. Araujo AQ and Silva MT: The HTLV-1 neurological complex. Lancet Neurol 2006;5:1068-1076.

5. Akizuki S, Nakazato O, Higuchi Y, et al.: Necropsy findings in HTLV-I associated myelopathy. Lancet 1987;1:156-157.

6. Cunha LCM, Tavares MC, Tierra-Criollo CJ, et al.: Contribution of galvanic vestibular stimulation for the diagnosis of HTLV-1-associated myelopathy/tropical spastic paraparesis. J Clin Neurol 2013;9:252-258.

7. Labanca L, Starling ALB, Carvalho SA, and Gonçalves DU: Dizziness, tinnitus and hearing loss related to HTLV-1 infection. Paper presented at the 30th International Congress of Audiology, March 28-April 1, 2010, São Paulo, Brazil.

8. Felipe L, Gonçalves DU, Santos MA, et al.: Vestibularevoked myogenic potential (VEMP) to evaluate cervical myelopathy in human T-cell lymphotropic virus type $\mathrm{i}$ infection. Spine 2008;33:1180-1184.

9. Felipe L, Kingma H, Lambertucci, JR, et al.: Testing the vestibular evoked myogenic potential (VEMP) to identify subclinical neurological alterations in different phases of human T-lymphotropic virus type 1 infection. Spine J 2012;12: 1362-1369.

10. Proietti FA, Carneiro-Proietti ABF, Catalan-Soares BC, and Murphy EL: Global epidemiology of HTLV-I infection and associated diseases. Oncogene 2005;24:6058-6068.

11. Allain JP, Stramer SL, Carneiro-Proietti ABF, et al.: Transfusion-transmitted infectious diseases. Biologicals 2009;37(2): 71-77.

12. Castro-Costa CM, Araújo AQC, Barreto MM, et al.: Proposal for diagnostic criteria of tropical spastic paraparesis/ HTLV-I-associated myelopathy (TSP/HAM). AIDS Res Hum Retroviruses 2006;22:931-935.

13. Kurtzke JF: Rating neurologic impairment in multiple sclerosis: An expanded disability status scale (EDSS). Neurology 1983;33:1444-1452.

14. Osame M: Review of WHO Kagoshima meeting and diagnostic guidelines for HAM/TSP. In: Human Retrovirology: HTLV (Blattner WA, ed.). Raven, New York, 1990, pp.191-197.

15. Munhoz MSL and Silva MLG : A avaliação audiológica no exame otoneurológico. In: Princípios de Otoneurologia (Ganança MM, Vieira RM, and Caovilla HH, eds.). Atheneu, São Paulo, 2000, pp. 7-22.

16. Maarsingh OR, Dros J, Weert HC, et al.: Development of a diagnostic protocol for dizziness in elderly patients in general practice: A Delphi procedure. BMC Fam Pract 2009;10:12.

17. Bronstein A and Thomas L: Tonturas-Diagnóstico e Tratamento-Uma Abordagem Prática. Revinter, Rio de Janeiro, 2009.

18. Rosengren SM, Welgampola MS, and Colebatch JG: Vestibular evoked myogenic potentials: Past, present and future. Clin Neurophysiol 2010;121:636-651.

19. Colebatch JG and Halmagyi GM: Vestibular evoked potentials in human neck muscles before and after unilateral vestibular deafferentation. Neurology 1992;42:1635-1636. 
20. Iles JF, Ali AS, and Savic G: Vestibular-evoked muscle responses in patients with spinal cord injury. Brain 2004; 127(7):1584-1592.

21. Felipe L, Kingma H, and Gonçalves DU: Potencial Evocado Miogênico Vestibular. Arquivos internacionais de otorrinolaringologia (Online) 2012; 16:103-107.

22. Isaradisaikul S, Strong DA, Moushey JM, et al.: Reliability of vestibular evoked myogenic potentials in healthy subjects. Otol Neurotol 2008;29:542-544.

23. Murofushi T, Shimizu K, Takegoshi H, and Cheng PW: Diagnostic value of prolonged latencies in the vestibular evoked myogenic potential. Arch Oto-laryngol Head Neck Surg 2001;127:1069-1072.

24. Gonçalves DU, Proietti FA, Barbosa-Stancioli, et al.: HTLV-1 associated myelopathy/tropical spastic paraparesis (HAM/TSP) inflammatory network. Inflamm. Allergy Drug Targets 2008;7:98-107.

25. Neuhauser HK, Von BM, Radtke A, et al.: Epidemiology of vestibular vertigo: A neurotologic survey of the general population. Neurology 2005;65:898-904.

26. Muto N, Shinomiya K, Komori H, et al.: Spinal cord monitoring of the ventral funiculus function. Analysis of spinal field potentials after galvanic vestibular stimulation. Spine 1995;20:2429-2434.
27. Felipe L, Gonçalves DU, Tavares MC, et al.: Vestibular evoked myogenic potential (VEMP) in the evaluation of schistosomal myeloradiculopathy. Am J Trop Med Hyg 2009;81:551-554.

28. Lambertucci JR, Silva LC, Andrade LM, et al.: Imaging techniques in the evaluation of morbidity in Schistosomiasis mansoni. Acta Trop 2008;108:209-217.

29. Hashimoto T, Uozumi T, and Tsuji S: Paraspinal motor evoked potentials by magnetic stimulation of the motor cortex. Neurology 2000;55:885-888.

30. Kaplan JE, Osame M, Kubota H, et al.: The risk of development of HTLV-1 associated myelopathy/tropical spastic paraparesis among persons infected with HTLV-1. J Acquir Immune Defic Syndr 1990;3:1096-1101.

Address correspondence to: Denise Utsch-Gonçalves Programa de Pós-Graduação em Infectologia e Medicina Tropical

Faculdade de Medicina da UFMG Av. Prof. Alfredo Balena, no. 190, sala 199 Belo Horizonte, Minas Gerais, CEP 30130-100

Brazil

E-mail: deniseg@medicina.ufmg.br 